\title{
Interferon-gamma release assays outcomes in healthy subjects following BNT162b2 mRNA COVID-19 vaccination
}

\author{
Ekaterina Kurteva $^{1,2}\left(\mathbb{C} \cdot\right.$ Georgi Vasilev $^{1,2}\left(\mathbb{D} \cdot\right.$ Kalina Tumangelova-Yuzeir $^{1,2}\left(\mathbb{C} \cdot\right.$ Irena Ivanova $^{3}(\mathbb{D} \cdot$ \\ Ekaterina Ivanova-Todorova ${ }^{1,2}$ ([) $\cdot$ Tsvetelina Velikova ${ }^{4,5}$ (1) Dobroslav Kyurkchiev ${ }^{1,2}$ (])
}

Received: 13 November 2021 / Accepted: 31 December 2021 / Published online: 20 January 2022

(C) The Author(s), under exclusive licence to Springer-Verlag GmbH Germany, part of Springer Nature 2022

\begin{abstract}
The pathogenesis of COVID-19 involves both humoral and cellular immunological responses, with cell-mediated immunity being discussed as the primary and most effective immune response to viral infection. It is supposed that COVID-19 vaccines also elicited effective cell immune response, and specifically IFN $\gamma$ secreted by SARS-CoV-2-specific T-helper 1 and Tcytotoxic cells. Using an interferon-gamma release assay (IGRA) test, we aimed to monitor cellular post-vaccination immunity in healthy subjects vaccinated with BNT162b2 mRNA COVID-19 vaccine (Comirnaty). We tested 37 healthcare workers (mean age 54.3 years, range 28-72, 22 females, 15 males) following COVID-19 mRNA COVID-19 vaccine and 15 healthy unvaccinated native persons as control subjects using QuantiFERON SARS-CoV-2 RUO test, performed approximately 1 month after vaccination. We also measured virus-neutralizing antibodies. Thirty-one out of 37 tested subjects had significantly raised levels of SARS-CoV-2 specific IFN $\gamma$ against SARS-CoV-2 Ag1 and Ag2 1 month following COVID-19 vaccination. In addition, we found a significant difference between the IFN $\gamma$ levels in fully vaccinated subjects and the control group $(p<0.01)$. We also found a substantial correlation $(r=0.9 ; p<0.01)$ between virus-neutralizing antibodies titers and IFN $\gamma$ concentrations released by T cells. We believe that IGRA tests are an excellent tool to assess the development of a post-vaccination immune response when immunized against SARS-CoV-2. However, IGRA-based tests should be performed within a few weeks following vaccination. Therefore, we can speculate that the application of these tests to assess long-term immune response is debatable.
\end{abstract}

Keywords COVID-19 $\cdot$ Interferon-gamma $\cdot$ Interferon-gamma release assay $\cdot$ SARS-CoV-2 $\cdot$ mRNA COVID-19 vaccine BNT162b2 vaccine $\cdot \mathrm{T}$ cytotoxic cells $\cdot \mathrm{T}$ helper cells $\cdot$ Cellular immune response $\cdot$ Immune memory

\section{Introduction}

Ekaterina Kurteva and Georgi Vasilev contributed equally to the paper and are co-first authors.

Tsvetelina Velikova

tsvelikova@medfac.mu-sofia.bg

1 Laboratory of Clinical Immunology, Unversity Hospital "St. Ivan Rilski", Akad. Ivan Geshov Blvd 15, 1431 Sofia, Bulgaria

2 Department of Clinical Immunology, Medical University of Sofia, Sofia, Bulgaria

3 Clinical Laboratory, "St. Ivan Rilski”, Akad. Ivan Geshov Blvd 15, 1431 Sofia, Bulgaria

4 Department of Clinical Immunology, University Hospital Lozenetz, Kozyak str., 1407 Sofia, Bulgaria

5 Sofia University St. Kliment Ohridski, Sofia, Bulgaria
The newly emerged SARS-CoV-2 virus is known for its numerous virulence factors, which together with the immunological processes following infection contribute to the organism's deterioration during coronavirus disease 19 (COVID-19). The pathogenesis of the illness involves both humoral and cellular immunological responses [1], with cellmediated immunity being discussed as the primary and most effective immune response to viral infection [2]. Immune memory and reinfection remain non-elucidated. However, great hopes were devoted to developing an efficient vaccination against the virus. Additionally, several therapeutic alternatives have been explored to identify an effective and specific therapy for COVID-19. At this point of knowledge, we may presume that the interaction between the SARSCoV-2 and the individual's immune system substantially 
impacts the disease's progression and prognosis, especially in patients with chronic diseases, including inflammatory and autoimmune $[3,4]$.

However, many concerns existed regarding the efficacy and safety of the novel COVID-19 vaccines, stepped in phase III clinical trials, especially in patients with autoimmune and inflammatory rheumatic diseases [5]. As a result, the first COVID-19 vaccine approved for emergency use by the US FDA on December 11, 2020, and by the EMA on December 21, 2020, is the mRNA-based vaccine Comirnaty developed in collaboration between Pfizer and BioNTech. Accordingly, the FDA and the EMA immediately authorized the Moderna COVID-19 vaccine for use on December 18, 2020, and January 06,2020 . Both vaccines use a single-stranded mRNA coding for the spike protein of the SARS-CoV-2.

There is a widespread misconception that the mechanism of action of the mRNA vaccines is new and unexplored; however, this is far from the case. As early as 1990, the first article was published describing immunization with mRNA in mice, which leads to intracellular translation of a protein. In 1992, the same process was demonstrated in rats [6]. Experiments in mice with mRNA vaccines encoding proteins of various viruses were published in 1993, 2012 (influenza virus), 2016 (rabies virus), 2013, 2016 (HIV), and 2017 (Zika virus). In pigs, such experiments were published in 2016 (rabies virus) and macaques in 2017 (Zika virus). The first clinical trials on the efficacy and safety of mRNA vaccines against the Zika virus were conducted in 101 healthy volunteers in 2017 [6].The same year, the results of a clinical trial examining the use of mRNA vaccines encoding influenza virus proteins in 23 participants were published. In 2018, the FDA and EMA approved the first Onpattro (Patisiran) mRNA vaccine for the treatment of Transthyretin-Related Familial Amyloid Polyneuropathy (TTR-FAP) in adults [6].

In 2020, Comirnaty was tested in a clinical trial involving 40,000 people, with a breakthrough (infection 2 weeks after the second dose) found in 8 people. Thus determines more than $95 \%$ efficiency [7].

The principle of action of the Comirnaty vaccine implicates the administration of non-infectious non-replicating mRNA. Understanding the mechanisms of action of COVID19 vaccines would help clinitians, especially immunologists and rheumatologists, in their practice. Moreover, investigating the elicited immune response, mainly cellular immune response, could be of practical benefit to measure the effectiveness of mRNA vaccines.

The mRNA molecule contains a $5^{\prime}$ cap, untranslated regions (UTR) before and after the genuine coding sequence, and a poly(A) tail at the $3^{\prime}$ end of the single strand. The mRNA carries a transcript encoding the Spike (S) protein of the SARS-CoV-2 virus [7]. As with all mRNA vaccines, this transcript encodes the corresponding protein using the myocyte translation apparatus $[6,7]$. mRNA got packaged in lipid nanoparticles (LPN), which degrade in endosomes, and thus mRNA is released and enters the cytoplasm [8]. However, it is essential to note that mRNA is not retained in the cytoplasm and does not enter the nucleus due to the exposition of RNase, which degrades it within a few days [7].

Meanwhile, in ribosomal complexes, mRNA translates, resulting in the formation of the $\mathrm{S}$ protein. The myocyte presents it on its surface along with the HLA-I molecule. Cytotoxic $\mathrm{T}(\mathrm{Tc})$ cells attack the corresponding complex. Tc cells secrete IFN $\gamma$ and act cytotoxic on the myocyte, allowing the S-protein to enter the extracellular space (Fig. 1).Intramuscular administration of mRNA/LNP results in a local inflammatory response in the muscle and the regional lymph nodes. Antigen-presenting cells (APCs) are involved, among which dendritic cells (DCs) play a significant role. They absorb the S protein and present it together with HLA-II, leading to recognition by $\mathrm{T}$ helper cells [9]. Thus, it targets both Th2 cells secreting IL-4 and mediating the activation of the humoral immune response, as well as Th1 cells secreting IFN $\gamma$ and determining the cellular immune response. Along with attracting APC, mRNA/LNP has a strong effect on the innate immune system. Genes encoding the synthesis of IL-1 $\beta$, MyD88, as well as gene complexes responsible for lipid uptake (LDLR), their processing (CTSL), and loading (TAP-2) are activated [9]. The mRNA itself is a typical pathogen-associated molecular pattern (PAMP) and activates endosomal (TLR-7) and cytoplasmic sensors (RIG-1) [6] (Fig. 1). However, we have to mention also that methylpseudouridine supports the linear structure of vaccine mRNA and thus, reduces immunogeneicity of vaccine because of TLR7 and RIG-1 [10].

The main effect of their activation is enhanced synthesis of type I interferons (IFNs-I). IFN-I activates the genes responsible for synthesizing $\mathrm{Mx}$ proteins and the chemokines CXCL-10 and CXCL-11 [9]. IFN-I also acts on DCs. They mature and express high levels of HLA-II and the costimulatory molecules CD80, CD86, all of which are associated with their ability to present both HLA-I and HLA-II molecules [6]. Moreover, under the influence of IFNs-I, the unique ability of DCs to cross-present is enhanced, allowing the antigenic presentation of the exogenous $\mathrm{S}$ peptide by HLA-I on Tc lymphocytes [9].

Intramuscular administration of mRNA/LNP leads to long-term production of the target protein, which determines the effective interaction in the germinal centers of the lymph nodes between $\mathrm{T}$ and B lymphocytes [6]. Tc cells and Th1 cells play a crucial role in the immune response after immunization with mRNA vaccines. While Th1 lymphocytes got primed upon presentation of the S protein by DCs, Tc cells got activated by cross-presentation by DCs and by recognizing the $\mathrm{S}$ protein expressed by myocytes (Fig. 1). All this allows us to try to answer the question "do I have cellular 


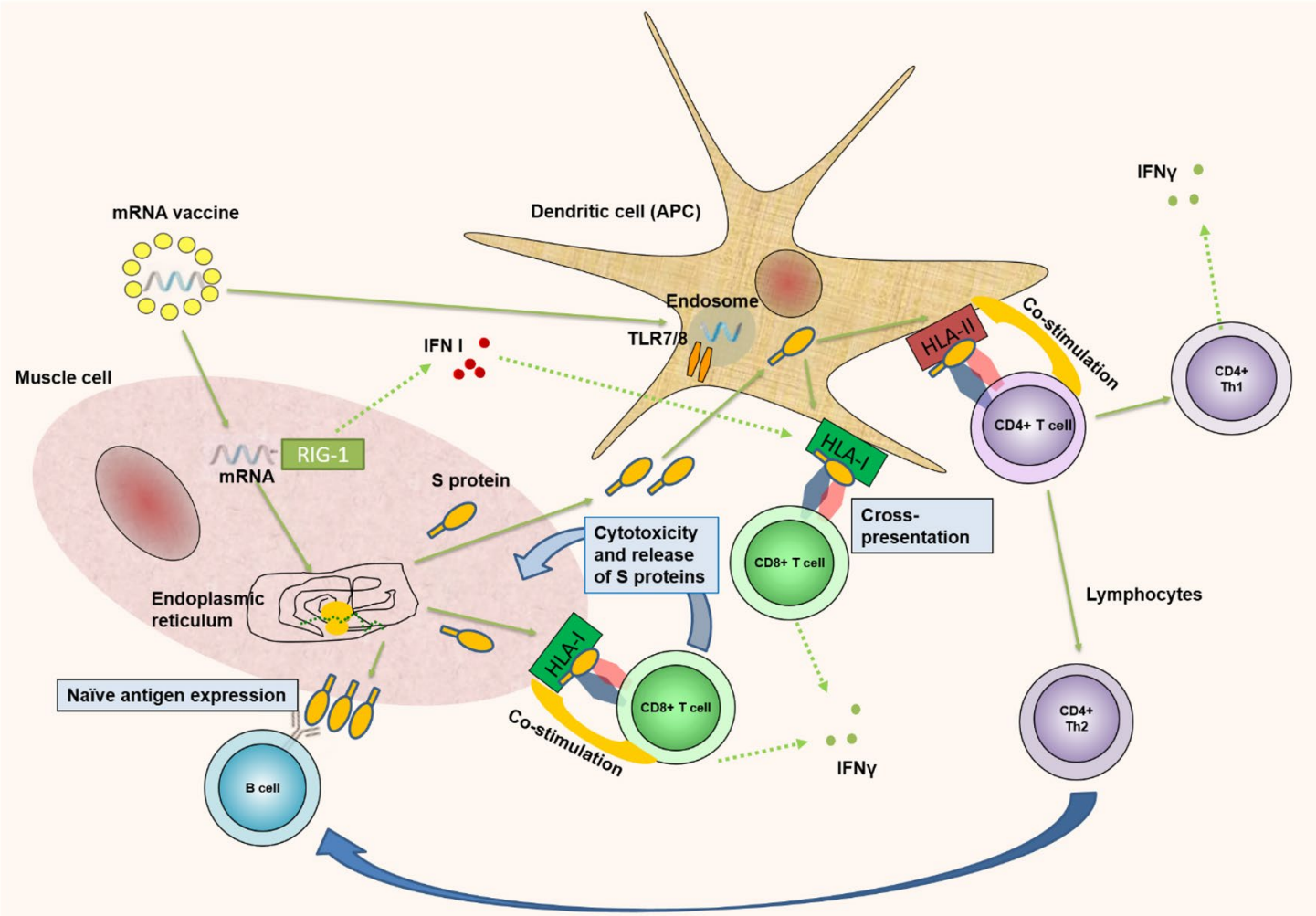

Fig. 1 Antigen-specific immune response after vaccination to SARSCOV-2. After intramuscular vaccination, mRNA/LNP(lipid nanoparticles) enters myocytes. There, LNPs are degrading to release the mRNA. It enters the endoplasmic reticulum, leading to translation of the mRNA molecule by the ribosomes and generation of the SARSCoV-2 spike protein. It has the fate of an endogenous antigen that is bound to the HLA-I molecule and presented on the cell surface. Recognition of Tcytotoxic (CD8 + Tc) cells leads to cytotoxic destruction of myocytes. A certain amount of the produced spike protein is secreted outside the myocytes. Spike protein enters the extracellular space. It is absorbed by dendritic cells (DCs) and acts as an exoge-

immune protection" by examining the levels of specifically secreted IFN $\gamma$ by these types of Th1 and Tc cells. Therefore, using an interferon-gamma release assay (IGRA) test, the present study aimed to monitor cellular postvaccination immunity in healthy subjects vaccinated with Comirnaty in January/February 2021.

\section{Methods}

\section{Study groups}

We tested 37 healthcare workers at University Hospital "XXX" (mean age 54.3 years, range 28-72, 22 females, 15 males) following COVID-19 vaccination with nous antigen, presented to T-helper 1 and T-helper $2(\mathrm{CD} 4+)$ cells. Myocyte mRNA activates interferon types I (IFN-I), which enhances the ability of DCs to cross-present exogenous HLA-I epitopes at Tc cells. We have to mention also that methylpseudouridine supports the linear structure of vaccine mRNA and thus, reduces immunogeneicity of vaccine because of TLR7 and RIG-1 [10]. Thick green arrowsproceed; green dot arrow-secretion; blue arrow-influence/help. $A P C$ antigen-presenting cell, TLR toll-like receptor, $H L A$ human leukocyte antigen, $I F N \gamma$ interferon-gamma, Th1 T helper cell 1 , Th2 T helper cell 2, RIG 1 retinoic acid-inducible gene I, IFN-I interferongamma type one

Pfizer-BioNTech COVID-19 vaccine to investigate cellmediated immune (CMI) response due to vaccination. In addition, we tested 15 healthy unvaccinated native persons as control subjects. The subjects received their vaccine in the period January/February 2021. The QuantiFERON SARS-CoV-2 RUO test was performed in April/March 2021, approximately 1 month after vaccination.

All subjects signed informed consent for inclusion in the study. The study design and protocol were conducted following the Declaration of Helsinki, previously approved by the Ethics Committee of the University Hospital "St. Ivan Rilski" (Ethics Approval Protocol Number 02/15.02.2021).

No sensitivity data were collected in our study. 


\section{Methods}

We used the QuantiFERON SARS-CoV-2 Blood Collection Tubes and QuantiFERON ELISA intended for Research Use Only (RUO) and not for diagnostic use. Diagnostic performances have not been established yet.

\section{Principle of the method}

The QuantiFERON SARS-CoV-2 RUO solution is an original combination of blood collection tubes containing innovative specific peptides formulated to stimulate lymphocytes in heparinized whole blood involved in cell-mediated immunity. Plasma from the stimulated samples can then be used for detection of IFN $\gamma$ using a simple ELISA.

We chose Control Set Blood Collection Tubes (QFN SARS Control BCTs), Cat No 626015, comprising QuantiFERON ${ }^{\circledR}$ Nil and QuantiFERON Mitogen BCTs intended to be used as negative and positive controls in conjunction with Starter Set Blood Collection Tubes (QFN SARS-CoV-2 BCTs), Cat No 626115, comprising two Antigen tubes, SARS-CoV-2 Ag1 and SARS-CoV-2 Ag2 using a combination of Spike protein peptides stimulating CD4 and CD8 T lymphocytes; and QuantiFERON ELISA (QFN) for detection of IFN $\gamma$ in plasma by enzyme-linked immunosorbent assay (ELISA), Cat No 626410 to perform our study.

\section{Procedure of the test}

The QuantiFERON SARS-CoV-2 Starter Set (QFN SARSCoV-2) Blood Collection Tubes uses a combination of antigens specific to SARS-CoV-2 to stimulate lymphocytes in heparinized whole blood involved in cell-mediated immunity. Plasma from the stimulated samples was used for the detection of IFN $\gamma$. Detection was measured using QuantiFERON ELISA. The QFN SARS-CoV-2 Blood Collection Tubes are not for use in diagnostic procedures.

The QuantiFERON ELISA (QFN ELISA) is a microplate coated with specific material for a human plasma sample (reported in International Units per $\mathrm{ml} ; \mathrm{IU} / \mathrm{ml}$ ). Analysis of results was performed using the QuantiFERON R\&D Analysis Software (RUO), Version 5.3.0

\section{Cut-off value}

Cut-off values have not been yet established, and research is ongoing. However, early data generated in independent studies (reference) suggest a cut-off between 0.15 and $0.2 \mathrm{IU} / \mathrm{ml}$ for both $\mathrm{Ag} 1$ and $\mathrm{Ag} 2$ tubes (Nil subtracted).

Virus-neutralizing antibodies in subjects were measured by Alinity i (Abbott, U.S.A.), CMIA (Chemiluminescent Microparticle Immuno Assay)-SARS-CoV-2 IgG II Quant assay. Values above $50 \mathrm{AU} / \mathrm{ml}$ are positive.

\section{Statistical analysis}

Statistical analysis of raw data was performed with GraphPad PRISM version 6 and RStudio. We used descriptive statistics, Mann-Whitney, ANOVA, correlation analysis. $p<0.05$ was considered significant.

Data collection was performed by the study's investigators according to the hospital's policies and good clinical practice. To avoid any potential sources of bias, data were collected and coded uniformly, taking into account and avoiding all sources of potential bias.

\section{Results}

Thirty-one out of 37 tested subjects had significantly raised levels of SARS-CoV-2 specific IFN $\gamma$ in Ag1 and $\mathrm{Ag} 2$ tubes (Nil subtracted) 1 month following COVID19 vaccination. Only six subjects (three men and three women) had a cut-off lower than $0.15 \mathrm{IU} / \mathrm{ml}$ for both $\mathrm{Ag} 1$ and Ag2 tubes (Table 1). In the control group, all 15 subjects had in their serum SARS-CoV-2 specific IFN $\gamma$ lower than $0.15 \mathrm{IU} / \mathrm{ml}$ for both $\mathrm{Ag} 1$ and $\mathrm{Ag} 2$ tubes.

We found a significant difference between the IFN $\gamma$ levels in fully vaccinated subjects and the control group (Fig. 2A). The Mean \pm SD and Median, Min-Max for the specific IFN $\gamma$ secretion, expressed in IU/ml, is also shown in Table 1.

Figure 2B presents the differences in IFN $\gamma$ levels in the negative control tube, $\mathrm{Ag} 1$ tube, $\mathrm{Ag} 2$ tube, and the mitogenic control tube for all vaccinated subjects enrolled in the study using a scatter plot. In addition, ascatter plot displaying the correlation $(r=0.9, p<0.01)$ between virus-neutralizing antibodies and IFN $\gamma$ differences measured by the QuantiFERON SARS-CoV-2 RUO test is shown in Fig. 2C.

\section{Discussion}

The present study examined T cell immunity 1 month after receiving the Comirnaty vaccine in healthy individuals (healthcare professionals, particularly). We designed our study this way because we took into account the kinetics of the immune response. Firstly followed the recommendations of the manufacturer stated that the Spike proteinspecific CD4 + and CD8 + Lymphocytes reach their peak levels 14 days following the second BNT162b2 mRNA COVID-19 vaccineand also we set a 1-month time limit to decrease the data variability. Also we took and extrapolated the previous finding made by Stephens and colleagues, that SARS-CoV-2 activates T cells in the first week of infection, 
Table 1 Mean \pm SD and Median, Min-Max for the specific IFN $\gamma$, IU/ml secretion of all 37 tested vaccinated subjects

\begin{tabular}{|c|c|c|c|c|}
\hline Patient no. & Negative control tube (IFN $\gamma . \mathrm{IU} / \mathrm{ml})$ & Ag1 tube (IFN $\gamma . \mathrm{IU} / \mathrm{ml})$ & $\mathrm{Ag} 2$ tube (IFN $\gamma . \mathrm{IU} / \mathrm{ml})$ & Mitogenic contol tube (IFN $\gamma \mathrm{IU} / \mathrm{ml})$ \\
\hline 1. & 0.01 & 2.54 & 3.03 & 10 \\
\hline 2. & 0 & 0.02 & 0.03 & 9.31 \\
\hline 3. & 0 & 0.62 & 1.29 & 10 \\
\hline 4. & 0 & 0.6 & 1.04 & 9.82 \\
\hline 5. & 0.08 & 0.73 & 1.21 & 10 \\
\hline 6. & 0.99 & 1.82 & 1.53 & 9.81 \\
\hline 7. & 0 & 0.42 & 0.99 & 10 \\
\hline 8. & 0.05 & 0.97 & 1.23 & 9.8 \\
\hline 9. & 0.01 & 3.12 & 3.44 & 10 \\
\hline 10. & 0 & 0.14 & 0.24 & 9.45 \\
\hline 11. & 0 & 0.14 & 0.28 & 9.65 \\
\hline 12. & 0 & 0.11 & 0.17 & 10 \\
\hline 13. & 0.02 & 0.22 & 0.18 & 9.52 \\
\hline 14. & 0.04 & 0.39 & 1.46 & 10 \\
\hline 15. & 0.18 & 1.82 & 1.88 & 10 \\
\hline 16. & 0 & 0.46 & 0.54 & 10 \\
\hline 17. & 0.03 & 0.18 & 0.31 & 10 \\
\hline 18. & 0.01 & 2.71 & 2.61 & 10 \\
\hline 19. & 0.01 & 0.38 & 0.53 & 10 \\
\hline 20. & 0 & 0.4 & 0.43 & 10 \\
\hline 21. & 0.02 & 0.19 & 0.11 & 10 \\
\hline 22. & 0.03 & 0.19 & 0.25 & 10 \\
\hline 23. & 0.06 & 0.03 & 0.05 & 9.69 \\
\hline 24. & 0.01 & 0.03 & 0.02 & 10 \\
\hline 25. & 0.1 & 0.73 & 1.15 & 10 \\
\hline 26. & 0.04 & 1.45 & 2.16 & 10 \\
\hline 27. & 0.01 & 2.38 & 2.02 & 10 \\
\hline 28. & 0.02 & 0.15 & 0.51 & 9.11 \\
\hline 29. & 0.03 & 0.45 & 0.33 & 10 \\
\hline 30. & 0.14 & 0.07 & 0.23 & 7.35 \\
\hline 31. & 0.01 & 0.12 & 0.17 & 7.93 \\
\hline 32. & 0 & 3.16 & 5.39 & 8.21 \\
\hline 33. & 0 & 0 & 0.02 & 8.48 \\
\hline 34. & 0.67 & 1.51 & 2.77 & 8.15 \\
\hline 35. & 0 & 0.1 & 0.1 & 8.59 \\
\hline 36. & 0.04 & 0.09 & 0.04 & 8.18 \\
\hline 37. & 0 & 0.92 & 2.32 & 9.18 \\
\hline Total & Mean $\pm S D=0.07 \pm 0.19$ & Mean $\pm \mathrm{SD}=0.8 \pm 0.94$ & Mean $\pm \mathrm{SD}=1.1 \pm 1.21$ & Mean $\pm \mathrm{SD}=9.5 \pm 0.74$ \\
\hline Total & Median, Min-Max $=0.01 ; 0-0.99$ & Median $=0.4 ; 0-3.16$ & Median $=0.53 ; 0.2-5.39$ & Median $=9.51 ; 7.35-10$ \\
\hline
\end{tabular}

and virus-specific memory CD4 + cells and CD8 + T cells reportedly peak within 2 weeks [11].

The subjects included in the study are predominantly rheumatologists and clinical immunologists from one of Bulgaria's leading university hospitals. From one point of view, they wished to check if they had a good level of protection against SARS-CoV-2 infection and reassure themselves that it is safe to meet their patients in person and provide them with the best quality health service. Along with that, the specialists could advise their patients strongly to get vaccinated for COVID-19, relying not only on the present evidence-based data but on their own experience concerning the postvaccine T-cellular immunity directed towards SARS-CoV-2.

The durability of this type of immunity has been the subject of much speculation and outright conspiracy theories [7, 12]. According to the literature, after suffering COVID-19, the development of immunity is observed for at least 1 year 

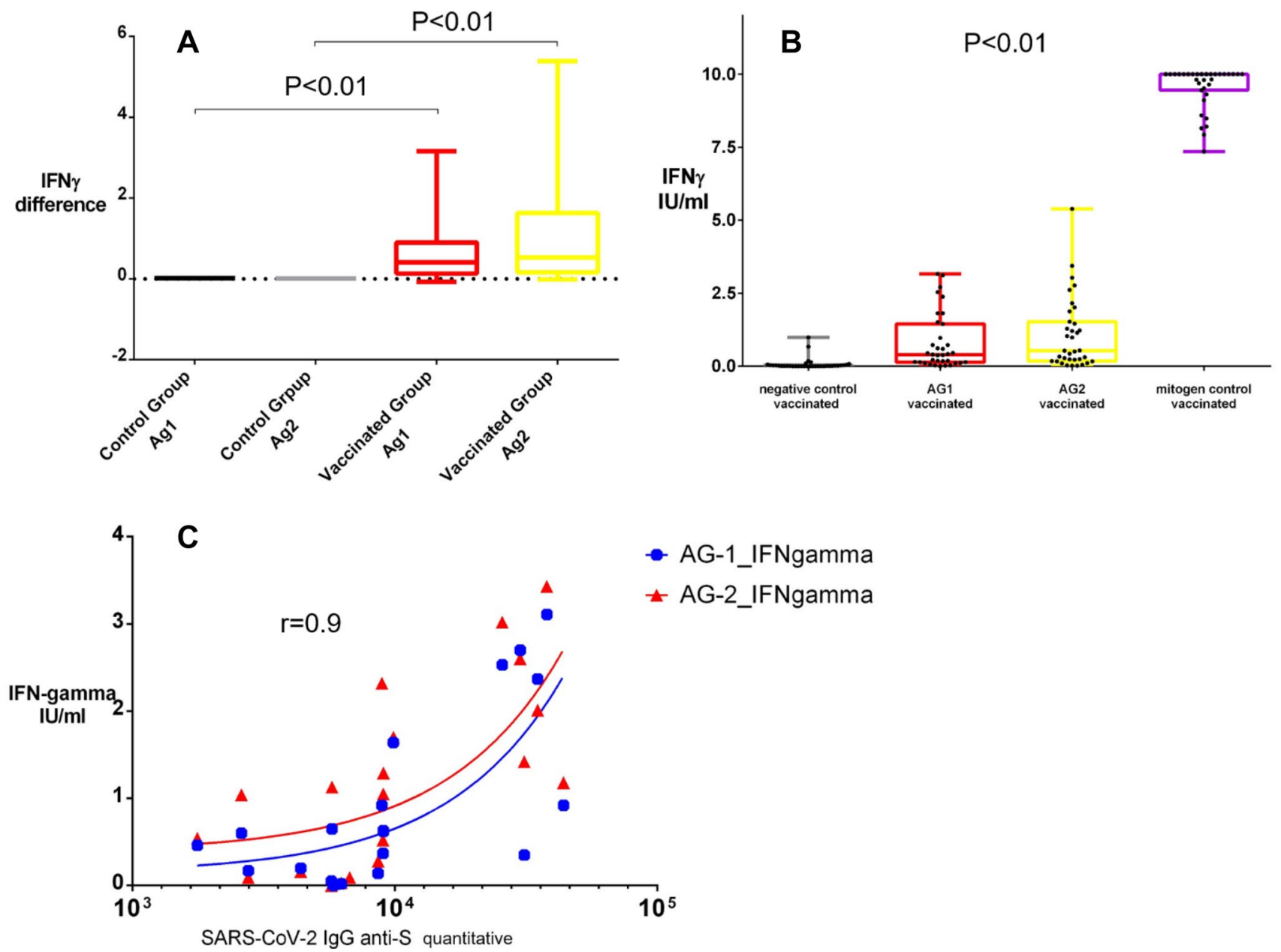

AG-1_IFNgamma

AG-2_IFNgamma

Fig. 2 Differences in SARS-CoV-2-specific IFN $\gamma$ levels between the native control group compared to the vaccinated group for both Ag1 and Ag2 tubes of the QuantiFERON SARS-CoV-2 RUO test (A); Scatter plots demonstrating the IFN $\gamma$ levels in the negative control

[13]. Therefore, it would be logical to achieve similar results after vaccination. Our data show that 6 out of 37 subjects did not show $\mathrm{T}$ cell immunity in peripheral blood. At the same time, none of these individuals became ill with COVID-19. We believe that the lack of cellular immune response in the peripheral blood in these individuals is related to several main factors.

First, we assumed that the cut-off value level of SARS$\mathrm{CoV}-2$ specific IFN- $\gamma$ was $0.15-0.2 \mathrm{IU} / \mathrm{ml}$. This value is still based on empirical observations [14] and cannot be considered definitive. It means that the validation of the kit is still in progress, and the cut-off value could be lower, turning some of the negative values into positive ones. The second factor is related to the time of the study. We obtained our results in a study conducted 1 month after the COVID-19 vaccination. Another study of 20 healthcare workers who received the Comirnaty vaccine with the same test showed that all the participants developed a cellular immune tube, Ag1 tube, Ag2 tube, and the mitogenic Contol tube for all vaccinated subjects enrolled in the $\operatorname{study}(\mathbf{B})$; Scatter plot displaying the correlation between antibodies and IFN $\gamma$ differences measured by the QuantiFERON SARS-CoV-2 RUO test (C)

response [15]. However, these results are based on a study performed in the days immediately following vaccination. The third factor is related to the second and focuses on the $\mathrm{T}$ cell immune response location. According to a recent study, 7 days after immunization with Comirnaty, plasmablasts with antigenic specificity against Spike protein's receptorbinding domain (RBD) were detected in the blood. They increase progressively until the third week, after which they begin to decrease.

On the other hand, the same type of plasmablasts are found in the germinal centers of the axial lymph nodes at least 15 weeks after vaccination, and their number does not decrease [16]. Similarly, antigen-specific T cells secreting IFN $\gamma$ should be observed in the blood shortly after the antigenic stimulus and then localized primarily in the lymph nodes (Fig. 3). Of course, these cells perform "patrolling" functions, periodically leaving the lymph nodes and entering the bloodstream. However, in our opinion, there is no 


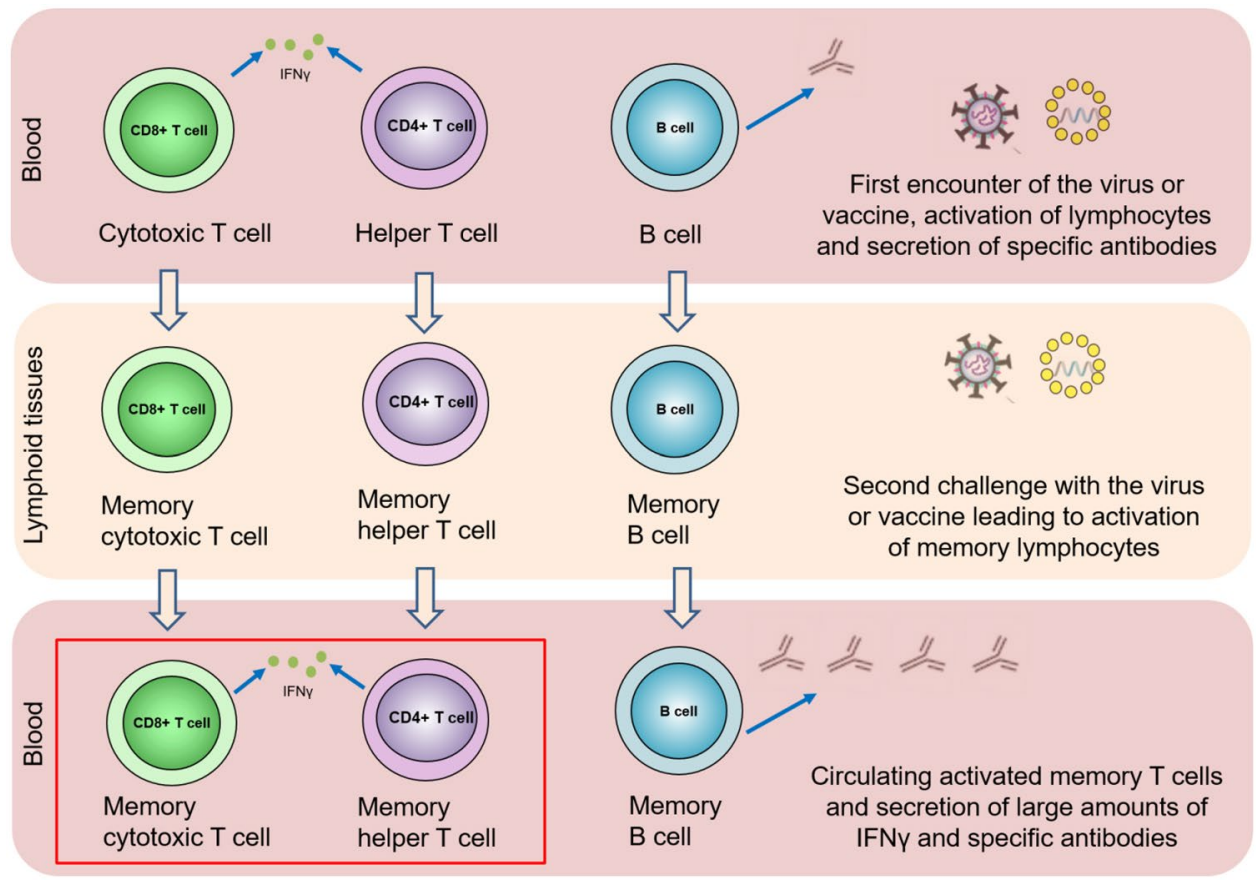

Fig. 3 Localization and migration of $\mathrm{T}$ cells in the course of the immune response. Immediately after the first encounter with the virusor vaccine, and sometime after acquiring immunity, immune cells (mainly $\mathrm{T}$ cells) specific to SARS-CoV-2 are present abundantly in the blood. Over time, specific memory cells got localized in the lymph nodes. Regularly, some of them "patrol" in the peripheral

guarantee that the test would be performed at this very moment to register them. Therefore, in the absence of an antigen stimulus, there is the likelihood that the IGRA test could not capture these cells (Fig. 3). It is essential to consider all these peculiarities of the immune response to avoid speculations such as "vaccines do not work".

In our opinion, the detection of antigen-specific $\mathrm{T}$ cells secreting IFN $\gamma$ in the blood provides definite certainty for the presence of a cellular immune response. Therefore, if the secretion of IFN $\gamma$ is in large quantities, we could speculate that there will be a recent encounter with SARS-CoV-2. However, we still can not interpret the test in cases with no cellular immune response in the peripheral blood. The major limitation of our study is the sample size. Additionally, we tested our subjects one moth after vaccination, thus, we cannot provide full information on what the IFNg levels would be on 3rd, 6th or 12th month after vaccination. However, we belive that limitations of the study are not fatal to draw our hypothesis and they are opportunities to inform future research.

To sum up, based on our results, studies from other study groups and the current knowledge of the immune responses against viruses and SARS-CoV-2 particularly, we believe that IGRA tests are an excellent tool to assess the development of a post-vaccination immune response when immunized against blood. SARS-CoV-2-specific $\mathrm{T}$ cells can be found again abundantly in the peripheral blood upon a new encounter with the virusor after revaccination. In the red quadrant-SARS-CoV-2-specific memory $\mathrm{T}$ cytotoxic cells that secrete IFN $\gamma$ are the cells that can be found by IGRA-based SARS-CoV-2 test in a relatively small time window after virus or vaccine re-challenge

SARS-CoV-2. Still, IGRA-based tests should be performed within a few weeks following vaccination. We can speculate that the application of these tests to assess long-term immune response is debatable.

Author contribution EK and GV contributed equally to this work. DK and EK were responsible for the conception and design of the manuscript. KT-Y and EI-T performed the methodology and data curation. GV analyzed the data, interpreted and validated the results with EK and EI-T. DK, EK, and TsV wrote the article. DK and TsV revised and edited the manuscript.

\section{Declarations}

Ethical approval The study design and protocol were conducted following the Declaration of Helsinki, previously approved by the Ethics Committee of the University Hospital "St. Ivan Rilski" (Ethics Approval Protocol Number 02/15.02.2021). No sensitivity data were collected in our study.

Informed consent All subjects signed informed consent for inclusion in the study. 


\section{References}

1. Velikova TV, Kotsev SV, Georgiev DS, Batselova HM (2020) Immunological aspects of COVID-19: What do we know? World J Biol Chem 11(2):14-29. https://doi.org/10.4331/wjbc.v11.i2.14

2. Velikova T (2021) Infection-acquired versus vaccine-induced immunity against COVID-19. Cent Asian J Med Hypotheses Ethics 2(1):29-35. https://doi.org/10.47316/cajmhe.2021.2.3.02

3. Raza HA, Sen P, Bhatti OA, Gupta L (2021) Sex hormones, autoimmunity and gender disparity in COVID-19. Rheumatol Int 41(8):1375-1386. https://doi.org/10.1007/s00296-021-04873-9

4. Ahmed S, Gasparyan AY, Zimba O (2021) Comorbidities in rheumatic diseases need special consideration during the COVID-19 pandemic. Rheumatol Int 41:243-256. https://doi.org/10.1007/ s00296-020-04764-5

5. Velikova T, Georgiev T (2021) SARS-CoV-2 vaccines and autoimmune diseases amidst the COVID-19 crisis. Rheumatol Int 41:509-518. https://doi.org/10.1007/s00296-021-04792-9

6. Pardi N, Hogan MJ, Porter FW, Weissman D (2018) mRNA vaccines-a new era in vaccinology. Nat Rev Drug Discov 17(4):261-279. https://doi.org/10.1038/nrd.2017.243

7. Löffler P (2021) Review: vaccine myth-buster-cleaning up with prejudices and dangerous misinformation. Front Immunol 10(12):663280. https://doi.org/10.3389/fimmu.2021.663280

8. van Riel D, de Wit E (2020) Next-generation vaccine platforms for COVID-19. Nat Mater 19(8):810-812. https://doi.org/10.1038/ s41563-020-0746-0

9. Liang F, Lindgren G, Lin A, Thompson EA, Ols S, Röhss J, John S, Hassett K, Yuzhakov O, Bahl K, Brito LA, Salter H, Ciaramella G, Loré K (2017) Efficient targeting and activation of antigenpresenting cells in vivo after modified mRNA vaccine administration in rhesus macaques. Mol Ther 25(12):2635-2647. https://doi. org/10.1016/j.ymthe.2017.08.006

10. Nance KD, Meier JL (2021) Modifications in an emergency: the role of N1-methylpseudouridine in COVID-19 vaccines. ACS
Cent Sci 7(5):748-756. https://doi.org/10.1021/acscentsci.1c001 97

11. Stephens DS, McElrath MJ (2020) COVID-19 and the path to immunity. JAMA 324(13):1279-1281. https://doi.org/10.1001/ jama.2020.16656

12. Gasparyan AY, Zimba O, Misra DP, Kitas GD (2020) Monitoring information flow on coronavirus disease 2019 (COVID-19). Mediterr J Rheumatol 31(Suppl 2):243-246. https://doi.org/10. 31138/mjr.31.3.243

13. Hansen CH, Michlmayr D, Gubbels SM, Mølbak K, Ethelberg S (2021) Assessment of protection against reinfection with SARS-CoV-2 among 4 million PCR-tested individuals in Denmark in 2020: a population-level observational study. Lancet 397(10280):1204-1212. https://doi.org/10.1016/S0140-6736(21) 00575-4

14. Van Praet JT, Vandecasteele S, De Roo A, De Vriese AS, Reynders M (2021) Humoral and cellular immunogenicity of the BNT162b2 mRNA Covid-19 vaccine in nursing home residents. Clin Infect Dis. https://doi.org/10.1093/cid/ciab300

15. Martinez-Gallo M, Esperalba-Esquerra J, Pujol-Borrell R (2021) T-cell responses as a correlate of COVID-19 vaccination. A pilot study in health care workers. MedRxiv. https://doi.org/10.1101/ 2021.03.31.21254472

16. Turner JS, O'Halloran JA, Kalaidina E, Kim W, Schmitz AJ, Zhou JQ, Lei T, Thapa M, Chen RE, Case JB, Amanat F, Rauseo AM, Haile A, Xie X, Klebert MK, Suessen T, Middleton WD, Shi PY, Krammer F, Teefey SA, Diamond MS, Presti RM, Ellebedy AH (2021) SARS-CoV-2 mRNA vaccines induce persistent human germinal centre responses. Nature 596(7870):109-113. https:// doi.org/10.1038/s41586-021-03738-2

Publisher's Note Springer Nature remains neutral with regard to jurisdictional claims in published maps and institutional affiliations. 